Research Paper

\title{
ECG-Guided Surveillance Technique in Cryoballoon Ablation for Paroxysmal and Persistent Atrial Fibrillation: A Strategy to Prevent From Phrenic Nerve Palsy
}

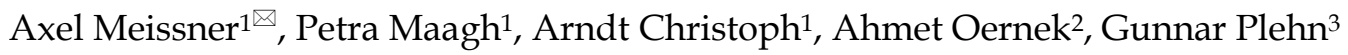

1. Department of Cardiology, Rhythmology and Internal Intensive Care, Klinikum Merheim, University Witten/Herdecke/Germany Ostmerheimer Str. 200, 51109 Cologne, Germany

2. Department of Diagnostic and Interventional Radiology, Berufsgenossenschaftliches Universitätsklinikum Bergmannsheil GmbH, Bürkle-de-la-Camp-Platz 1,44789 Bochum

3. Department of Cardiology and Angiology, Johanniter-Krankenhaus Rheinhausen GmbH, Kreuzacker 1-7, 47228 Duisburg, Germany

$\triangle$ Corresponding author: Axel Meissner, Ruhr-University Bochum, Universitätsstraße 150, 44801 Bochum. Mail: axel.meissner@rub.de

(C) Ivyspring International Publisher. Reproduction is permitted for personal, noncommercial use, provided that the article is in whole, unmodified, and properly cited. See http://ivyspring.com/terms for terms and conditions.

Received: 2015.11.11; Accepted: 2016.04.20; Published: 2016.05.10

\begin{abstract}
Aims: Phrenic nerve palsy (PNP) is still a cause for concern in Cryoballoon ablation (CBA) procedures. New surveillance techniques, such as invasive registration of the compound motor action potential (CMAP), have been thought to prevent the occurrence of PNP. The present study investigates the impact of CMAP surveillance via an alternative and non-invasive ECG-conduction technique during $\mathrm{CBA}$.

Methods: PVI with CBA was performed in 166 patients suffering from AF. Diaphragmal contraction was monitored by abdominal hands-on observation in Observation Group I; Observation Group II was treated using additional ECG-conduction, as a means of modified CMAP surveillance method. During the ablation of the right superior and inferior pulmonary veins, the upper extremities lead I was newly adjusted between the inferior sternum and the right chest, thereby recording the maximum CMAP. The CMAP in the above-mentioned ECG leads was continuously observed in a semi-quantitative manner.
\end{abstract}

Results: PNP was observed in 10 (6\%) patients in total. In Observation Group I, 6 out of 61 (9.8\%) demonstrated PNP. In Observation Group II a significant decrease of PNP could be demonstrated $(p<0,001)$ and occurred in 4 out of 105 patients $(3.8 \%)$. While three patients from Observation Group I left the EP lap with an ongoing PNP, none of the patients in Observation Group II had persistent PNP outside of the EP lab.

Conclusion: The present study demonstrates that additional ECG-conduction, used as modified CMAP surveillance, is an easy, effective and helpful additional safety measure to prevent PNP in CBA.

Key words: Atrial Fibrillation; Pulmonary Vein Isolation; Cryoballoon Ablation, Phrenic Nerve palsy, Compound Motor Action Potential

\section{Introduction}

\section{Cryoballon Ablation of Atrial Fibrillation}

Atrial fibrillation is the most commonly sustained arrhythmia and has been treated using conventional radiofrequency ablation in a point-by-point fashion for years [1,2]. However, due to emerging technology, several single-device techniques have been establishing themselves as more reasonable alternatives in the therapy of AF. Within the scope of single-device techniques, CBA has played a prominent role and is a well-established technique 
for ablation of PAF and less common for ablation of PerAF [3-5].

The acute procedural efficacy, long-term effects, as well as peri-procedural complications observed so far have been previously described by several studies [6-9]. Complications mainly occurring within the context of CBA involve minor remote damage. However, serious or persistent clinical injury may occur in the form of PNP [10]. While pericardial tamponade and cerebral embolism are typical complications of the PVI procedure itself - and also mainly observed in non-single device PVI techniques - PNP is notably and solely characteristic of CBA and sometimes as well in laser balloon ablation.

\section{Strategies to Avoid Phrenic Nerve Palsy}

There are some strategies for avoiding PNP during the ablation of the right-sided pulmonary veins. A deep CB position should always be avoided, as the anatomical distance to the PN becomes shortened (Figure 1). In the same manner, and often observed in the latter anatomical position, very low temperatures of between -55 and -60 degrees should not be exceeded during ablation of the RSPV and RIPV. As these practices may be helpful for patients to avoid the occurrence of PNP, they are not suitable in cases of incomplete PVI. Although PNP should obviously be avoided, successful ablation remains the main goal. A further strategy to prevent PNP is ongoing fluoroscopic observation during ablation of the RSPV and RIPV. Many practitioners combine these techniques with continuous PN stimulation in the vena cava superior and hands-on diaphragmal contraction surveillance. Apart from the fluoroscopic stress, the loss of diaphragmal contraction is quite often not induced by true PNP, but rather by dislocation of the PN stimulation catheter. The attempt to correct the phrenic nerve stimulation catheter during ongoing ablation may take too long to preserve the functional integrity of the PN. The time intervals between the loss of phrenic nerve capture, the loss of diaphragmal contractility and the immediate interruption of cryothermal freezing is the main issue in this scenario.

\section{New Surveillance Techniques of Phrenic Nerve Integrity}

In addition to these considerations, further techniques to prevent PNP have been reported by Franceschi and coworkers [11], and studied in firstand second-generation $C B$ procedures $[12,13]$. Using a quadripolar catheter positioned in the subdiaphragmatic vein, a modified diaphragmatic compound motor action potential (CMAP) was recorded and introduced as an additional safety measure in CBA. When a 30\% drop in the CMAP was observed, the ablation procedure was immediately interrupted and subsequently carried out further in a modified manner. None of the patients suffered any complications nor was any PNP observed. To simplify the method and to translate the CMAP of the phrenic muscle to the surface, we readjusted the extremities lead I electrodes of right and left arm, thereby recording the maximum amount of CMAP (Figure 2). The action potential in the named ECG leads was continuously observed in a semi-quantitative manner during PVI in a beat-to-beat analysis. We hypothesize that the introduction of this safety measure to the CBA procedure may lead to a significant reduction of PNP in a prospective real world ablation scenario.

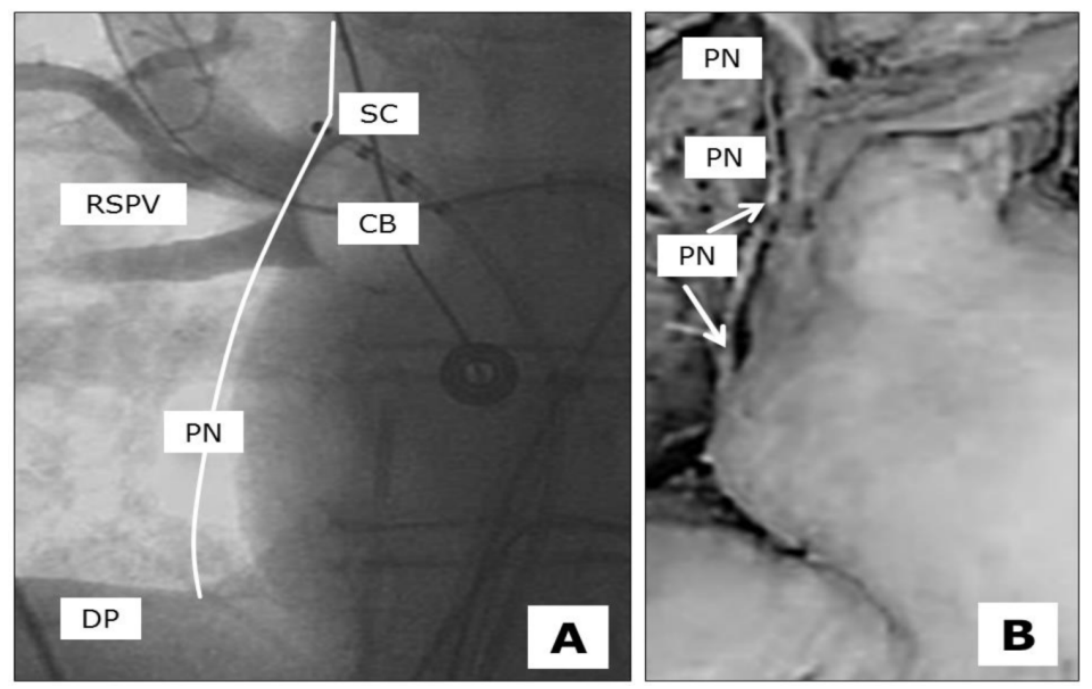

Figure 1: Anatomical proximity between the underlying Phrenic Nerve (PN), Right Superior Pulmonary Vein (RSPV), Cryoballon 28 mm (CB) and the stimulation catheter (SC) at the optimal stimulation point located close to the upper edge of the pulmonary vein, diaphragm (DP). A, fluoroscopy in the cathlab, theoretical course of the PN close to the ablation site, B, anatomícal preparation with isolated PN. 


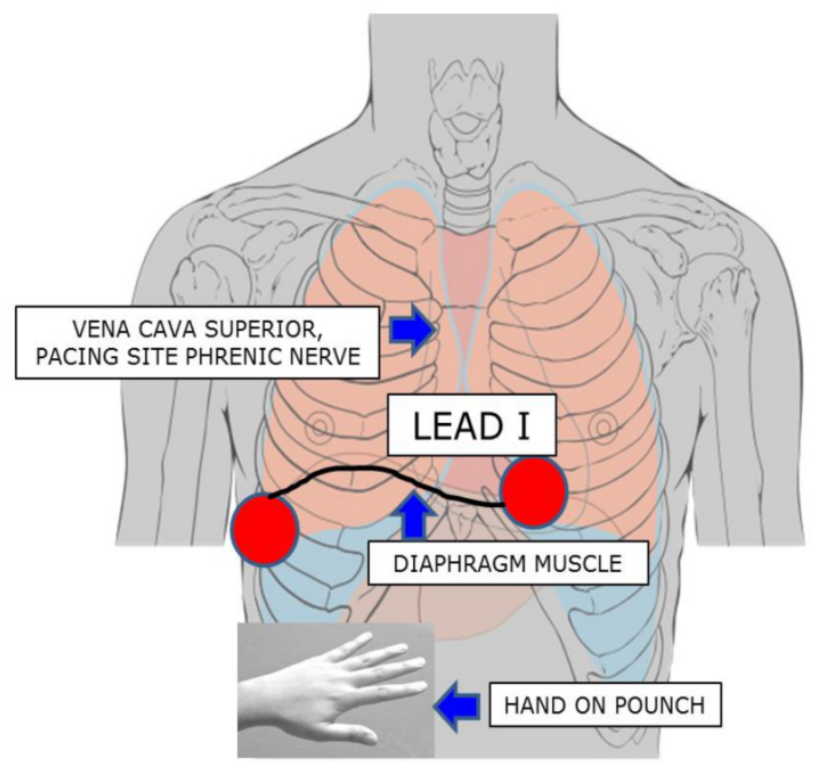

Figure 2: Phrenic Nerve Stimulation by placing the coronary sinus catheter in the lower vena cava inferior. The upper extremities lead I was newly adjusted: electrode left arm was placed $5 \mathrm{~cm}$ above the xyphoid process at the left margin of the inferior sternum, electrode right arm was placed on the xyphoid level in the right front axillary line, thereby recording the maximum diaphragmatic compound motor action potential. Simultaneous hand on pounch in the subdiaphragmal abdominal area for supervision of muscle contraction.

\section{Methods}

\section{Demographic Data}

Between 07/2012 - 07/2015, this prospective study examined 166 patients $(62.9 \pm 11.4$ years old) with highly symptomatic AF and resistant to specific antiarrhythmic medication. The majority of this patient group suffered from PAF (109 patients $/ 65.7 \%$ ), while fewer were in the early stages of PerAF (57 patients/34.3\%). Out of this patient group, 92 patients $(55.4 \%)$ were men (average age $62.9 \pm 11.4$ ) and had suffered from AF on average for 33 months (32.6 \pm 46 months). The main risk factor in this patient group was arterial hypertension; 136 patients $(81.9 \%)$ were on medication and received at least one or more specific pills in addition to the antiarrhythmic drugs, mainly beta-blockers. The cohort included patients from $\mathrm{CHADS}^{2} \mathrm{VASC}$ risk factor scores of between 0-8, dominated by the low- and medium-grade CHADS scores of three, two and one (49 patients /29.5\%, 47 patients $/ 28.5 \%$ and 30 patients $/ 18.1 \%$, respectively).

\section{Pre-Ablation Preparation}

All 166 patients included in the study were properly informed and provided prior written consent. The study subjects met the following inclusion criteria: a history of highly symptomatic PAF (min. of 1 episode/week) or PerAF despite treatment with one or more antiarrhythmic drugs.
Two out of the three investigators present were trained in $C B$ technique trained and performed the procedures. Exclusion criteria were as follows: an left atrium diameter of $\geq 55 \mathrm{~mm}$, severe left ventricular hypertrophy ( $\mathrm{LV}$ wall thickness of $\geq 15 \mathrm{~mm}$ ), LA thrombus, prior stroke and/or current decompensated heart failure.

Prior to the ablation procedure, all patients had undergone a trans-thoracic and trans-esophageal echocardiography as well as a standardized computed tomography scan with three- dimensional reconstruction of the PV and the left atrium. The patients were kept on their daily medications and anticoagulation was alleviated but not interrupted with Coumadin, so that the procedure could be performed with an International Normalised Ratio of between 1.8-2.2. In contrast to this proceeding, patients being treated with new oral anticoagulants (NOAC`s, Dabigatran, Rivaroxaban, Apixaban), stopped taking their medication for at least 2 days prior to the procedure - this time was bridged by administering standard or fractionated heparin.

\section{Catheter Ablation Procedure}

All procedures were performed with patients being under deep sedation using midazolam and fentanyl bolus injections as well as a continuous infusion of Propofol (1\%). Vital parameters, such as heart rate, blood pressure and oxygen saturation, were continuously monitored throughout the entire procedure. Three catheters were deployed during the procedure: A decapolar coronary sinus catheter for pacing purposes and anatomical orientation prior to the transseptal puncture, a 10-polar circular catheter (Achieve catheter $\AA$; Medtronic, Pointe-Claire, Canada) was inserted into the $\mathrm{CB}$ catheter (Arctic Front $\mathrm{TM} \otimes$ and Arctic Front Advanced $\mathrm{TM} \AA$, Medtronic Cryocath, Pointe-Claire, Canada) for mapping and observation purposes, and the $C B$ catheter itself. Likewise, from the left groin, we performed an arterial puncture for the purpose of vital parameter observation and activated clotting time surveillance. The CBA was performed as described in previous studies $[7,8]$.

The freezing cycle was started- using an ArcticFrontTM ${ }^{\circledR}$ (generally consisting of two $300 \mathrm{~s}$ freezes) and an Arctic Front AdvancedTM ${ }^{\circledR}$ (generally consisting of two $240 \mathrm{~s}$ freezes). In-situ temperature was continuously monitored using a sensor at the proximal part of the $\mathrm{CB}$. Temperatures lower than -60 degrees Celsius or a dislocation of the $\mathrm{CB}$ resulted in the immediate interruption of the energy delivery in all PVs. 


\section{Phrenic Nerve}

When ablating the right-sided PV, further safety measures were applied. In both ablation groups, the coronary sinus catheter was withdrawn from its original position and placed in the superior vein cava. Phrenic nerve capture was observed by constantly pacing with a $10 \mathrm{~V}$ amplitude and $2.9 \mathrm{~ms}$ pulse width close to the PV and could be monitored visually or by hands-on monitoring of stomach diaphragm contractility. In the case of PNP or weakening, freezing was immediately terminated applying the double stop technique.

In addition to the described approach, we applied a further safety measure to prevent patients in the second ablation group from PNP from May 2012 onward (Arctic Front Advanced TM®). Therefore, during ablation of the RSPV and RIPV, the upper extremities lead I was newly adjusted between the inferior sternum and the right chest, thereby recording the maximum diaphragmatic compound motor action potential (Figure 2). Electrode left arm was placed $5 \mathrm{~cm}$ above the xyphoid process at the left margin of the inferior sternum, electrode right arm was placed on the xyphoid level in the right front axillary line. The amplitude of CMAP was continuously recorded on lead I and observed in a semi-quantitative manner during PVI in a beat-to beat-analysis (Figure 3). Interference with more of $\geq 30 \%$ ECG amplitude decrease or complete loss of the ECG amplitude led to an immediate interruption of the freeze. In the case of capture loss or interference of

Table 1: Patient characteristics of the entire study population

\begin{tabular}{|l|c|c|}
\hline Patient characteristics & N & $\%$ \\
\hline Age (years) & & $\mathbf{6 3} \pm \mathbf{1 1}$ \\
\hline Sex (male) & 92 & $\mathbf{5 5 , 4}$ \\
\hline AF-type & & \\
\hline PAF, paroxysmal AF & 109 & $\mathbf{6 5 , 7}$ \\
\hline CAF, persistent AF & 57 & $\mathbf{3 4 , 3}$ \\
\hline AF duration & & $\mathbf{3 2} \mathbf{6} \pm \mathbf{1 1} \mathbf{4}$ \\
\hline LA diameter & & $\mathbf{4 , 8} \mathbf{2}, \mathbf{5}$ \\
\hline Reduced Ejection fraction & 12 & $\mathbf{7 , 2}$ \\
\hline Hypertension & 136 & $\mathbf{8 1 , 9}$ \\
\hline Diabetes & 27 & $\mathbf{1 6 , 3}$ \\
\hline Hyperlipoproteinamia & 118 & $\mathbf{7 1 , 1}$ \\
\hline Coronary artery disease & 42 & $\mathbf{2 5 , 3}$ \\
\hline Hypertensive Heart Cardiomyopathie & 32 & $\mathbf{1 9 , 4}$ \\
\hline Failed antiarrhymic drug & 85 & $\mathbf{5 1 , 2}$ \\
\hline Class I Agents & 48 & $\mathbf{2 8 , 9}$ \\
\hline Amiodarone & 22 & $\mathbf{1 3 , 3}$ \\
\hline Sotalol & 3 & $\mathbf{1 , 8}$ \\
\hline Dronedarone & 12 & $\mathbf{7 , 2}$ \\
\hline Ablation tool & & $\mathbf{2 7 , 1}$ \\
\hline First generation cryoballon & 45 & $\mathbf{7 2 , 9}$ \\
\hline Second generation cryoballon & 121 & \\
\hline
\end{tabular}

$\mathrm{AF}=$ Atrial fibrillation, $\mathrm{LA}=$ Left atrium. the ECG amplitude, PNS was continuously conducted until PN recovery could be observed. In the meantime, the current coronary sinus catheter position was compared with the original position, and when dislocated, readjusted to its original position if necessary.

After each freeze, PV conduction was re-evaluated by positioning the Achive catheter at the same location within the PV as before the ablation. Based on the CBA, the Achive was pulled back as proximally as possible until it dropped into the LA. The PV was considered to be eisolated when the following indications were observed: (1) All PV potentials could be eliminated and (2) The exit block could be confirmed by pacing from inside the vein distally to the ablation line. After a waiting period of 30 min. after the last PVI, persistence of the conduction block was re-checked with the Achieve catheter in all veins.

\section{Statistical analysis}

All data was presented as mean \pm standard deviation (SD). SPSS 22.0 software package was used for statistical analysis. Student's t-test and Chi-square test were used to determine the statistical significance of differences of numerical and categorical data. A p value of $<0.05$ was statistically significant.

\section{Results}

\section{Demographic and Ablation Data}

In all 166 patients, the procedure could be completed and none of the patients experienced any serious complications. 45 patients $(27.1 \%)$ were ablated with the first generation $\mathrm{CB}$, while in the majority of 121 patients $(72.9 \%)$, the procedure was performed with the second generation CB. One third of all patients (61 patients/36.6\%), belong to Group 1 - with conventional PN surveillance and the majority of which were ablated using the first generation $\mathrm{CB}$ - while the remaining patients (Group 2, 105 patients $/ 63.4 \%$ ) were exclusively ablated with the second generation $\mathrm{CB}$ and experienced the extended surveillance protocol.

The clinical baseline-characteristics of the entire study population are summarized in Table 1.

Complete PVI could be achieved in 162 of the 166 patients (97.2\%); in 4 patients, one of four veins could not be isolated and had to be re-ablated with radiofrequency energy. In the LSPV, we applied an average of 2.3 freezes ( \pm 0.82 , ranging from 1 to 7 ) with an 
average application time of 293 seconds ( \pm 135 , ranging from 180 to 1000) and an average temperature of 51 degrees Celsius ( \pm 5.9 , ranging from 36 to 65 ). In the left-inferior pulmonary vein (LIPV), we applied an average of 2.1 freezes ( \pm 0.61 ranging from 1 to 5$)$ with an average application time of 292 seconds $( \pm 152$, ranging from 140 to 1000) and an average temperature of 46 degrees Celsius ( \pm 9.3 , ranging from 37 to 69$)$. In the RSPV, we applied an average of 2.1 freezes $( \pm 0.67$, ranging from 1 to 5) with an average application time of 289 seconds ( \pm 139 , ranging from 210 to 1000) and an average temperature of 49 degrees Celsius $( \pm 8.5$, ranging from 38 to 68). In the RIPV, we applied an average of 1.9 freezes $( \pm 0.56$, ranging from 1 to 4$)$ with an average application time of 251 seconds ( \pm 80 , ranging from 0 to 750 ) and an average temperature of 44 degrees Celsius ( \pm 11 , ranging from 33 to 73 ).

\section{Phrenic Nerve Palsy}

PNP occurred in 10 out of 166 patients (6.0\%) and appeared as either a transient or an ongoing phenomenon. When transient, the diaphragm contractility was abrogated for only moments (a few seconds), minutes or at the most for less than 1 hour, and recovery could be demonstrated by the resumption of phrenic nerve capture due to stimulation. This was observed in 7 out of 10 patients $(70 \%)$. Three patients $(30 \%)$ demonstrated prolonged PNP and left the operating table with a complete loss of diaphragm contractility. Clinical symptoms were fatigue, dyspnea either while at rest or under stress and intermittent right-sided chest pains. Two patients recovered from PNP within 6 months and 1 patient partially did so after one year of having the procedure. Patients with PNP were of the average age of 64 (ranging from 48-79) and PNP was mainly observed in the RSPV (8 patients) and less often in the RIPV (2 patients) with an average temperature of -50 degrees Celsius after 1.6 ablation freezes. The demographic and technical ablation data of the 10 patients with PNP are summarised in Table 2.

Of the 10 patients with PNP we observed more PNP in the Observation Group I, despite the fairly smaller number of procedures. Despite immediate freeze interruption in the occurrence of PNP or when a reduction of diaphragm contractility was observed, the damage was obvious. Even the hands-on technique, which instantly reacts to a loss of contractility, could not prevent PNP in all cases.

In the fairly larger Observation Group II (consisting of 105 patients), we observed only 4 patients with PNP (equaling 3.8\% of Observation Group II, Figure 6). Due to the ECG conduction modified in comparison to the conventional Study Group I, the amplitude of the QRS in lead I could be easily observed, as demonstrated in Figure 3, 4 and 5. During ablation of the RSPV and RIPV, the ECG-amplitude was continuously observed and semi-quantitatively assessed in a beat-to-beat analysis. As can be seen in Figure 5, a reduction of more than $50 \%$ of the amplitude could be noticed prior to the loss of contractility, indicating an infringement of the phrenic nerve. The immediate interruption of the freeze led to a significant reduction in PN disturbance. Those patients sustaining PN injury with this technique recovered immediately, subsequently demonstrating rapid growth in the ECG amplitude. Ongoing PNP was not observed in this study group. Typically, the reduction of ECG amplitude occurred prior to the loss of diaphragm contractility hereby proving itself as a more sensitive observation tool for $\mathrm{CB}$ ablation techniques.

By comparing the two observation groups, a significant difference was determined in the frequency of PNP $(p<0.001)$. In relation to the different number of patients ablated in both groups fairly more clinical events occurred in Observation Group I than in Observation Group II, indicating that extended ECG monitoring can reduce clinical events, even despite the application of the more effective and aggressive ablation system, see Figure 6. Despite the complications with PNP, no other relevant complications occurred in our observation groups.

Table 2: All patients with PNP during cryoballon ablation, demographic and technical data: Sex; Age; Location, Temperature, Time and Freeze in which PNP occured. Resolution from PNP intraprocedural.

\begin{tabular}{|c|c|c|c|c|c|c|c|}
\hline Pat. & Sex & Age & Location & Temperature & $\begin{array}{l}\text { Time to } \\
\text { PNP }\end{array}$ & Freeze & Resolution \\
\hline \#1 & $f$ & 66 & RSPV & -40 & 150 & 2 & $j$ \\
\hline$\# 2$ & $f$ & 76 & RSPV & -52 & 125 & 1 & $n$ \\
\hline \#3 & m & 48 & RSPV & -48 & 160 & 2 & j \\
\hline \#4 & m & 72 & RSPV & -48 & 110 & 1 & $\mathbf{j}$ \\
\hline \#5 & f & 75 & RIPV & -60 & 230 & 1 & $\mathrm{n}$ \\
\hline$\# 6$ & m & 51 & RSPV & -58 & 120 & 1 & $\mathrm{n}$ \\
\hline$\# 7$ & m & 64 & RSPV & -45 & 50 & 1 & j \\
\hline$\# 8$ & $f$ & 72 & RSPV & -43 & 180 & 2 & $j$ \\
\hline \#9 & $f$ & 79 & RSPV & -57 & 200 & 1 & $j$ \\
\hline$\# 10$ & m & 57 & RIPV & -48 & 190 & 3 & $j$ \\
\hline
\end{tabular}




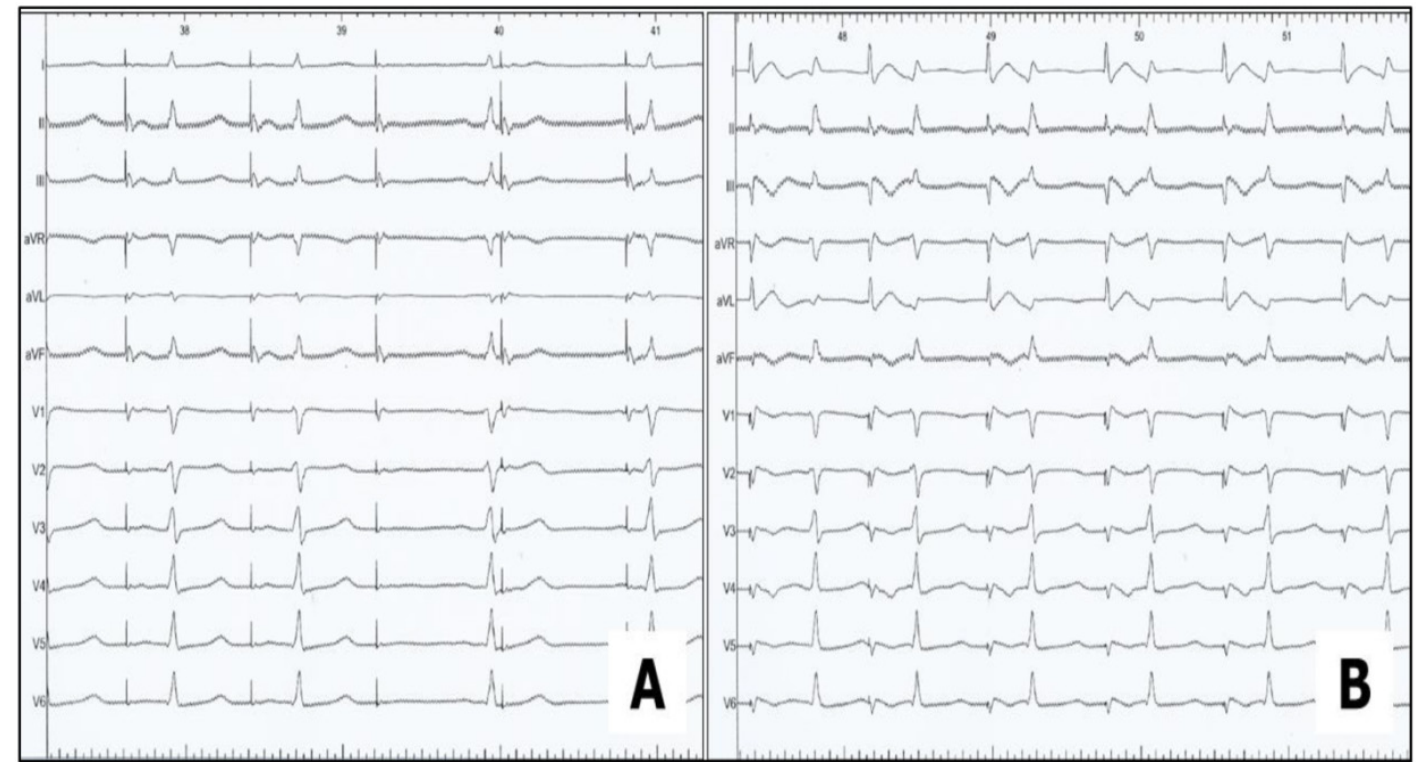

Figure 3: Phrenic Nerve Stimulation without (A) and with (B) modified ECG-arrangement, the upper extremities lead I was newly adjusted between the inferior sternum and the right chest, thereby recording the maximum diaphragmatic compound motor action potential (CMAP).

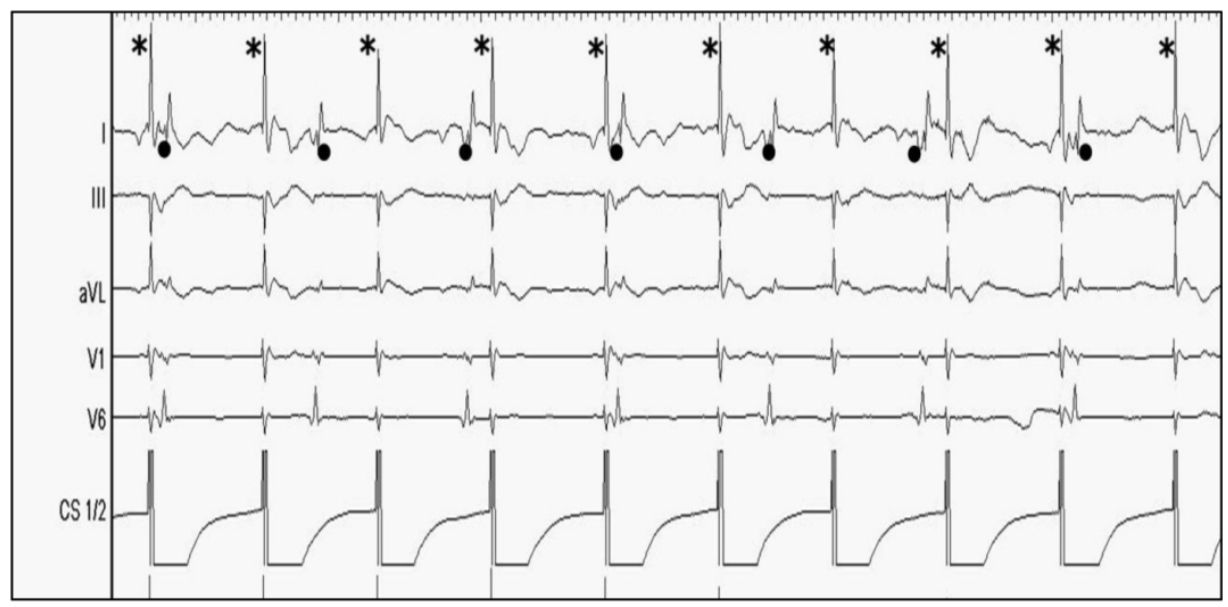

Figure 4: Ongoing Phrenic Nerve Stimulation via coronary sinus catheter pacing of the distal electrodes CS1/2 during ablation of the RSPV and RIPV. The dominant pacing amplitude (marked with an asterisk*) is easy to distinguish from the consecutive QRS-complexes (marked as point $\bullet$ ) in sinus rhythm. From the top to the bottom: Surface ECG leads: I, II, aVL, V1, V6. Endo-cardiac signals: Coronary sinus CS 1/2.

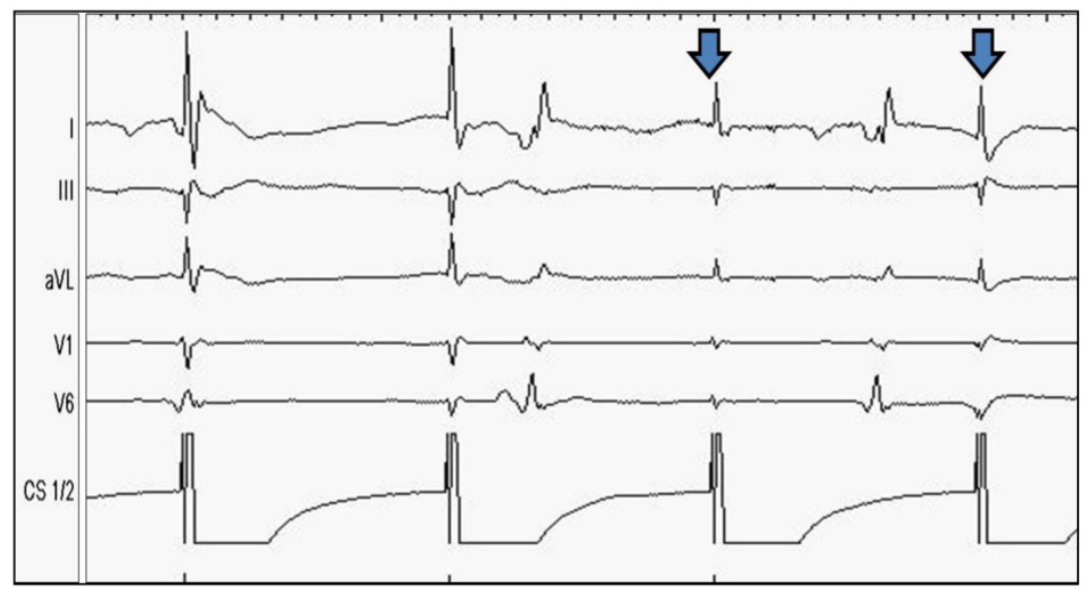

Figure 5: Prior to the loss of contractility, a slight decrease of ECG-amplitude can be noticed; the contractility of the diaphragm is still preserved at this point. From the top to the bottom: Surface ECG leads: I, II, aVL, V1, V6. Endo-cardiac signals: Coronary sinus CS 1/2. 


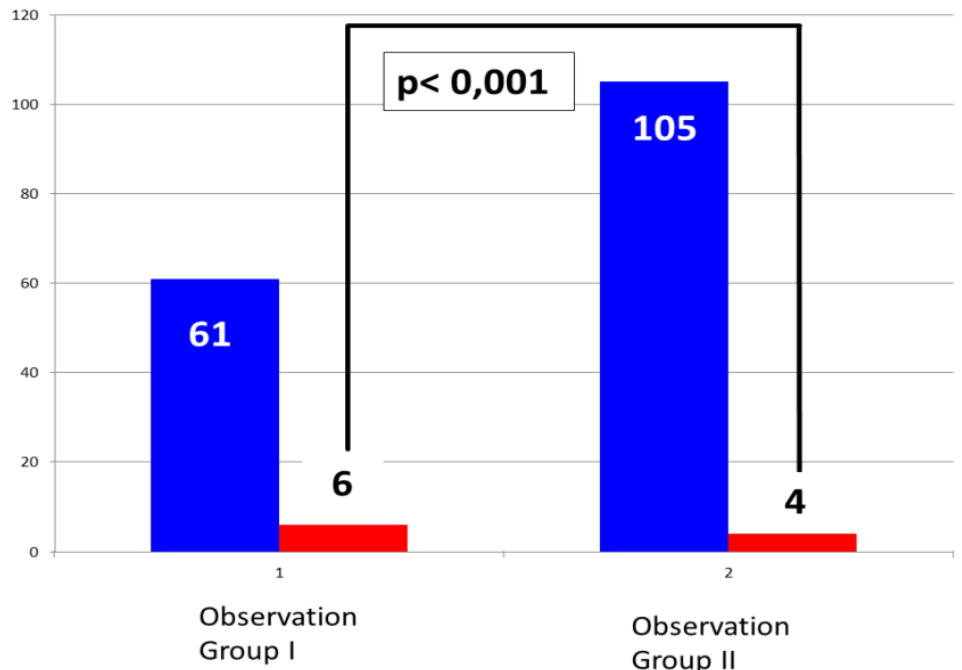

Figure 6: Portion of phrenic nerve palsy in Group 1 without CMAP surveillance (1) and Group II with CMAP surveillance (2).

\section{Discussion}

\section{General Considerations}

The interventional ablation therapy of AF is actually dominated by two approaches - the conventional radiofrequency ablation technique in a point-by-point fashion and the $\mathrm{CB}$ ablation technique, which has become the most widely accepted, single-device techniques worldwide. Both, major and minor complications are still grounds for concern and have been described by several studies and registries over the last decade [2, 5-10]. There are some reasons why the $\mathrm{CB}$ technique has become one of the most accepted single-device techniques. Besides its promising data for acute and long- term ablation success, the technique is quite simple and easy to use. Acute and long-term outcomes of catheter ablation of $\mathrm{AF}$ using $\mathrm{CB}$ technology versus open-irrigated radiofrequency are comparable and show no significant differences [14].

\section{Phrenic Nerve Palsy in Cryoballoon Ablation}

Apart from the common AF complication profile, PNP has been described as the only main problem relatively specific to and quite frequently observed in $\mathrm{CB}$ ablation procedures, ranging between $5-10 \%$ [8-10]. The reason for this phenomenon is the close physiological position of the ablation site and the course of the phrenic nerve (next and subjacent to the right-sided PV, Figure 1) [15-17]. Although virtually always reversible over time, cases of permanent PNP have been reported [10].

Earlier studies have described the importance of diaphragmatic electromyography monitoring during $\mathrm{CB}$ ablation for the prevention of diaphragm muscle contractility loss due to PNP. Franceschi et al. therefore recorded a compound motor action potential (CMAP) using a quadripolar catheter positioned in a sub-diaphragmatic hepatic vein during the $C B$ procedure [11-13]. In smaller study series, CMAP recording seems safe and potentially even helpful to prevent PNP. Miyazaki et al. recently evaluated diaphragmatic electromyograms from surface electrodes (CMAPsuf) and the sub-diaphragmatic hepatic veins (CMAPabd) during PV antrum isolation. They obtained stable CMAPs from the surface in most patients; both surface and invasive CMAP recordings seem to be an alternative or complementary method for the prevention of PNP [18]. Lakhani and coworkers stated in a smaller study series, that recording of CMAP amplitude on a modified lead I is reliable and could predict from PNP in patients undergoing CBA for PAF and PerAF [19].

\section{Present Study}

The present study investigates the impact of CMAP surveillance via an alternative ECG-conduction tool during the CBA of the right-sided PVs. Both $\mathrm{CB}$ generations were applied, whereby in the larger Study Group II with only advanced surveillance techniques, the novel Arctic Front Advance CB was exclusively applied. Fürnkranz et al. reported improved procedural efficacy of pulmonary vein isolation in ablation using a novel second-generation cryoballoon [20]. Recently, Cassado et al. reported a significant increase of right-sided PNP, seeming to occur in a significantly larger number of patients having undergone second-generation CBA [9]. Surprisingly, the authors observed PNP not only in the RSPV, as reported in the majority of previous studies, but also in the RIPV within the scope of their study. Anatomical studies conducted on cadavers have shown that the distance 
from the right PN to the RSPV is much shorter when compared to the RIPV (2-3 $\mathrm{mm}$ vs $10-12 \mathrm{~mm}$ ) [15]. Cassado et al. speculated that this might be due to the more extensive area of freezing, reaching the tip of the balloon. The increase of ablation effect by using the second generation $\mathrm{CB}$ has been confirmed by other study groups as well as by our present study [9]. Fürnkranz et al. recently compared both generations of CBs. The use of the second generation $\mathrm{CB}$ resulted in a significantly higher rate of single-shot PVI, shorter procedural durations, and lower fluoroscopy exposure times within the scope of their study. In contrast to Cassado's findings, Fürnkranz did not observe an increase of PNP when using the novel CB generation; the total amount of PNP was low compared to the overall observed occurrence of this complication [21].

Based on such recently published data as mentioned above, the main findings of our study are as follows: (1) PNP was the main complication in a consecutive series of patients using the first and the second generation $\mathrm{CB}$ and it occurred in $6 \%$ of all patients. (2) The introduction of a novel surveillance tool leads to a significant decrease of PNP, despite being a more effective ablation technique. ECG monitoring is non-invasive and easy to handle (3) PNP occurs predominantly in the RSPV, but also may occur in the RIPV, as did occur in a quarter of cases of our study. (4) PNP mainly occurred around -50 degrees Celsius - half of the patients demonstrating PNP did not do so before the second freeze in both right-sided PVs. (5) In all cases, PNP was a reversible complication, with the vast majority of patients having recovered during the procedure.

Thus, the observation of PNP - both in the RSPV, but also in the RIPV - as the most frequent complication in $\mathrm{CB}$ ablation procedures using firstand second-generation CBs can be confirmed by our study and is in line with the current literature. Ablation in a real world scenario confirms the findings of other studies conducted in that advanced surveillance techniques are helpful in the prevention of PNP. This study observed PNP in $6 \%$ of all patients. This finding is comparable to current literature. Instead of a PNP increase when using the more effective ablation technique, we observed a significantly lower complication rate. PNP is a prompt and unpredictable event in $\mathrm{CB}$ procedures, which may not only lead to an interruption of the freeze in the vein, but may in some cases even result in procedure interruption. Recovery time and the uncertainty regarding the progress of the procedure make PNP a troublesome occurrence.

With the use of CMAP observation using an alternative ECG-conduction technique, we could prevent patients from imminent PN trauma by decreasing the ECG amplitude prior to the reduction of diaphragm contractility in a semi-quantitative manner. This beat-to-beat analysis significantly reduced abrupt loss of diaphragm contractility as freezes could be stopped prior to an onset of PN injury. The technique is simple to use, can be conducted by a medical technical assistant and does not prolong the procedure. The observation of the ECG amplitude is easy to manage and offers more safety for both the patient and examiner alike. We therefore strongly propose the routine use of this observational technique in every $\mathrm{CB}$ ablation procedure.

\section{Study limitations}

There are some study limitations to be acknowledged. Firstly, the present study is a single center, observational, non-randomized clinical trial. It describes the first experiences with the modified CMAP technique in a real world scenario. Secondly, despite the fact that all examiners were very experienced operators in regard to $C B$ procedures, they continually learned how to better handle complication occurrences over time. This may have led to a bias concerning the second study group - with fewer PNP observations. Thirdly, the first and the second generation $\mathrm{CB}$ were used in the first observation group, in the observation group 2 PVI was performed using the second generation $\mathrm{CB}$ exclusively. As differences in the incidence of PNP have been reported earlier between both $\mathrm{CB}$ generations, it should be considered that this might represent a bias. Fourthly, the sample size of study group one was relatively small. Nevertheless, a significant difference could be determined between both study cohorts. Certainly, the observation of significantly fewer PNP occurrences when using the modified CMAP technique should be confirmed within the scope of larger study cohorts. Fifthly, the observation of ECG modifications was conducted in a semi-quantitative manner. Nevertheless, the discriminatory power was obviously strong enough to prevent patients from experiencing PNP, thereby leading to a significant reduction of the complication rate in the second study group.

\section{Abbreviations}
AF: Atrial Fibrillation
CB: Cryoballoon
CBA: Cryoballoon Ablation
CMAP: Compound Motor Action Potential
PAF: Paroxysmal Atrial Fibrillation
PerAF: Persistent Atrial Fibrillation
PN: Phrenic Nerve 
PNS: Phrenic Nerve Stimulation

PNP: Phrenic Nerve Palsy

PV: Pulmonary Vein

PVI: Pulmonary Vein Isolation

RSPV: Right Superior Pulmonary Vein

RIPV: Right Inferior Pulmonary Vein

\section{Competing Interests}

The authors have declared that no competing interest exists.

\section{References}

[1] Haissaguerre M, Shah DC, Jais P, Hocini M, Yamane T, Deisenhofer I, Chauvin M, Garrigue S, Clementy J. Electrophysiological breakthroughs from the left atrium to the pulmonary veins. Circulation 2000; 14:2463-2465.

[2] Calkins H, Kuck KH, Cappato R, Brugada J, Camm AJ, Chen SA et al. 2012 HRS/EHRA/ECAS expert consensus statement on catheter and surgical ablation of atrial fibrillation: recommendations for patient selection, procedural techniques, patient management and follow-up, definitions, endpoints, and research trial design. Europace 2012; 14:528-606.

[3] Neumann T, Vogt J, Schumacher B, Dorszewski A, Kuniss M, Neuser H et al. Circumferential pulmonary vein isolation with the cryoballoon technique results from a prospective 3-center study. J Am Coll Cardiol 2008; 52:273-8.

[4] Malmborg H, Lönnerholm S, Blomström P, Blomström-Lundqvist C. Early experience with the cryoablation balloon procedure for the treatment of atrial fibrillation by an experienced radiofrequency catheter ablation center. Europace. 2013 May 22.

[5] Andrade JG, Khairy P, Guerra PG, et al. Efficacy and safety of cryoballoon ablation for atrial fibrillation: a systematic review of published studies. Heart Rhythm 2011;8: 1444-1451.

[6] Guiot A, Savouré A, Godin B, Anselme F. Collateral nervous damages after cryoballoon pulmonary vein isolation. J Cardiovasc Electrophysiol 2012; 23:346-351.

[7] Saitoh Y, Irfan G, Ciconte G, Mugnai G, Sieira J, DI Giovanni G, Baltogiannis G, Conte G, Hünük B, Ströker E, Velagić V, Overeinder I, DE Asmundis C, Chierchia GB, Brugada P. Persistence of Phrenic Nerve Palsy Following $28 \mathrm{~mm}$ Cryoballoon Ablation: A Four-Year Single Center Experience. Pacing Clin Electrophysiol. 2015 Jul; 38(7):807-14.

[8] Kühne M, Knecht S, Altmann D, et al. Phrenic nerve palsy during ablation of atrial fibrillation using a $28-\mathrm{mm}$ cryoballoon catheter: predictors and prevention. J Interv Card Electrophysiol 2013; 36:47-54.

[9] Casado-Arroyo $\mathrm{R}$, Chierchia GB, Conte $\mathrm{G}$, Levinstein $\mathrm{M}$, Sieira J, Rodriguez-Mañero M, di Giovanni G, Baltogiannis Y, Wauters K, de Asmundis C, Sarkozy A, Brugada P. Phrenic nerve paralysis during cryoballoon ablation for atrial fibrillation: a comparison between the first- and second-generation balloon. Heart Rhythm. 2013 Sep;10(9): 1318-24.

[10] Mugnai G, de Asmundis C, Ciconte G, Irfan G, Saitoh Y, Velagic V, Ströker E, Wauters $K$, Hünük B, Brugada P, Chierchia GB. Incidence and characteristics of complications in the setting of second-generation cryoballoon ablation: A large single-center study of 500 consecutive patients. Heart Rhythm. Heart Rhythm. 2015 Jul; 12(7):1476-82.

[11] Franceschi F, Dubuc M, Guerra PG, Khairy P. Phrenic nerve monitoring with diaphragmatic electromyography during cryoballoon ablation for atrial fibrillation: the first human application. Heart Rhythm 2011; 8:1068-1071.

[12] Franceschi F, Koutbi L, Mancini J, Attarian S, Prevôt S, Deharo JC. Novel electromyographic monitoring technique for prevention of right phrenic nerve palsy during cryoballoon ablation. Circ Arrhythm Electrophysiol. 2013 Dec; 6(6):1109-14

[13] Franceschi F, Koutbi L, Gitenay E, Hourdain J, Maille B, Trévisan L, Deharo JC. Electromyographic monitoring for prevention of phrenic nerve palsy in second-generation cryoballoon procedures. Circ Arrhythm Electrophysiol. 2015 Apr; 8(2):303-7

[14] Aryana A, Singh SM, Kowalski M, Pujara DK, Cohen AI, Singh SK, Aleong RG, Banker RS, Fuenzalida CE, Prager N, Bowers MR, d'Avila A, O'Neill PG. Acute and Long-term Outcomes of Catheter Ablation of Atrial Fibrillation Using the Second-Generation Cryoballoon Versus Open-Irrigated Radiofrequency: A Multicenter Experience. J Cardiovasc Electrophysiol. 2015 Aug; 26(8):832-9.

[15] Sanchez-Quintana D, Cabrera JA, Climent V, et al. How close are the phrenic nerves to cardiac structures? Implications for cardiac interventionalists. J Cardiovasc Electrophysiol 2005; 16:309-313.

[16] Schmidt B, Chun KR, Ouyang F, et al. Three-dimensional reconstruction of the anatomic course of the right phrenic nerve in humans by pace mapping. Heart Rhythm 2008; 5:1120-1126.

[17] Andrade JG, Dubuc M, Guerra PG, et al. The biophysics and biomechanics of cryoballoon ablation. Pacing Clin Electrophysiol 2012; 35:1162-1168.

[18] Miyazaki S, Ichihara N, Taniguchi H, Hachiya H, Nakamura H, Usui E, Kanaji Y, Takagi T, Iwasawa J, Kuroi A, Hirao K, Iesaka Y. Evaluation of diaphragmatic electromyograms in radiofrequency ablation of atrial fibrillation: prospective study comparing different monitoring techniques. J Cardiovasc Electrophysiol. 2015 Mar; 26(3):260-5.

[19] Lakhani M, Saiful F, Parikh V, Goyal N, Bekheit S, Kowalski M. Recordings of diaphragmatic electromyograms during cryoballoon ablation for atrial fibrillation accurately predict phrenic nerve injury. Heart Rhythm 2014; 11(3):369-74

[20] Fürnkranz A, Bordignon S, Schmidt B, et al. Improved procedural efficacy of pulmonary vein isolation using the novel second-generation cryoballoon. J Cardiovasc Electrophysiol 2013; 24(5):492-497.

[21] Fürnkranz A, Bordignon S, Schmidt B, Perrotta L, Dugo D, De Lazzari M, Schulte-Hahn B, Nowak B, Chun JK. Incidence and characteristics of phrenic nerve palsy following pulmonary vein isolation with the second-generation as compared with the first-generation cryoballoon in 360 consecutive patients. Europace. 2015 Apr; 17(4):574-8. 